UCRHEP-T334

April 2002

\title{
Neutrino Mass from Triplet and Doublet Scalars at the TeV Scale
}

\author{
Ernest Ma \\ Physics Department, University of California, Riverside, California 92521
}

\begin{abstract}
If the minimal standard model of particle interactions is extended to include a scalar triplet with lepton number $L=-2$ and a scalar doublet with $L=-1$, neutrino masses $m_{\nu} \sim \mu_{12}^{4} v^{2} / M^{5} \sim 10^{-2} \mathrm{eV}$ is possible, where $v \sim 10^{2} \mathrm{GeV}$ is the electroweak symmetry breaking scale, $M \sim 1 \mathrm{TeV}$ is the typical mass of the new scalars, and $\mu_{12} \sim 1 \mathrm{GeV}$ is a soft lepton-number-violating parameter.
\end{abstract}


In the minimal standard model of particle interactions, neutrinos are massless, but if new interactions exist at a higher scale, then they may become massive through the unique effective dimension-five operator [1]

$$
\mathcal{L}_{\text {eff }}=\frac{f_{i j}}{\Lambda} L_{i} L_{j} \Phi \Phi,
$$

where $L_{i}=\left(\nu_{i}, l_{i}\right)_{L}$ is the usual left-handed lepton doublet, $\Phi=\left(\phi^{+}, \phi^{0}\right)$ is the usual scalar Higgs doublet, and $\Lambda$ is an effective large mass. There are three tree-level realizations [2] of this operator: (I) the canonical seesaw mechanism [3] using one heavy right-handed neutrino $N_{R}$ for each $\nu_{i}$; (II) the addition of a heavy Higgs triplet $\left(\xi^{++}, \xi^{+}, \xi^{0}\right)$ which couples to $L_{i} L_{j}$ directly [4, 包; and (III) the replacement of $N_{R}$ with $\left(\Sigma^{+}, \Sigma^{0}, \Sigma^{-}\right)_{R}$ [6, 7]. If the mass of $N$ or $\xi$ or $\Sigma$ is very large, then each realization is the same as any other at low energies, because the only observable effect would be the appearance of small Majorana neutrino masses.

It has recently been pointed out that simple extensions of the above minimal scenarios for neutrino mass are possible for which the scale of new physics may be only a few $\mathrm{TeV}$ and thus be observable at future accelerators. There are two specific proposals: (A) the Higgs triplet $\xi$ may be only a few $\mathrm{TeV}$, whose decay into two leptons would map out all elements of the neutrino mass matrix [8]; and (B) the fermion singlets $N_{R}$ as well as a second Higgs doublet $\eta=\left(\eta^{+}, \eta^{0}\right)$ may be only a few $\mathrm{TeV}$ [9].

In (A), the notion of lepton-number violation as a distance effect from the separation of our brane from another in the context of large extra dimensions is invoked to explain the smallness of the trilinear scalar coupling of $\xi$ to $\Phi \Phi$. In (B), there is no need to consider large extra dimensions. Instead, the effective operator of Eq. (1) is suppressed because $\Phi$ is replaced with $\eta$, which has a naturally small vacuum expectation value. In this note, the two mechanisms are synthesized so that there is a Higgs triplet $\xi$ from (A), and a Higgs doublet $\eta$ from (B), but no $N_{R}$. Neutrino masses come from $\xi$ (which is assigned lepton number $L=-2$ ), and its interaction with $\eta$ (which has $L=-1$ ). The smallness of $m_{\nu}$ comes from 
the soft breaking of $L$ in the scalar sector, with the result

$$
m_{\nu} \sim \frac{\mu_{12}^{4} v^{2}}{M^{5}} \sim 10^{-2} \mathrm{eV}
$$

where $v \sim 10^{2} \mathrm{GeV}$ is the electroweak symmetry breaking scale, $M \sim 1 \mathrm{TeV}$ is the typical mass of the new scalars, and $\mu_{12} \sim 1 \mathrm{GeV}$ is a soft lepton-number-violating parameter. This model requires neither large extra dimensions nor $N_{R}$ to obtain naturally small Majorana neutrino masses, and have verifiable experimental consequences at the $\mathrm{TeV}$ scale.

In the Higgs triplet model, $\xi$ couples to leptons according to

$$
\mathcal{L}_{Y}=f_{i j}\left[\xi^{0} \nu_{i} \nu_{j}+\xi^{+}\left(\nu_{i} l_{j}+l_{i} \nu_{j}\right) / \sqrt{2}+\xi^{++} l_{i} l_{j}\right]+H . c .,
$$

resulting in $\left(m_{\nu}\right)_{i j}=2 f_{i j}\left\langle\xi^{0}\right\rangle$. Therefore, in order to explain why neutrino masses are so small, a natural mechanism for $\left\langle\xi^{0}\right\rangle$ to be small is needed. Consider now the Higgs sector consisting of the usual standard-model doublet $\Phi$ (with $L=0$ ), a second doublet $\eta$ (with $L=-1$ ), and a triplet $\xi($ with $L=-2)$. Let

$$
\Delta \equiv\left(\begin{array}{cc}
\xi^{+} / \sqrt{2} & \xi^{++} \\
\xi^{0} & -\xi^{+} / \sqrt{2}
\end{array}\right),
$$

then the most general $L$-conserving Higgs potential is given by

$$
\begin{aligned}
V & =m_{1}^{2} \Phi^{\dagger} \Phi+m_{2}^{2} \eta^{\dagger} \eta+m_{3}^{2} \operatorname{Tr} \Delta^{\dagger} \Delta \\
& +\frac{1}{2} \lambda_{1}\left(\Phi^{\dagger} \Phi\right)^{2}+\frac{1}{2} \lambda_{2}\left(\eta^{\dagger} \eta\right)^{2}+\frac{1}{2} \lambda_{3}\left(\operatorname{Tr} \Delta^{\dagger} \Delta\right)^{2}+\frac{1}{2} \lambda_{4}\left(\operatorname{Tr} \Delta^{\dagger} \Delta^{\dagger}\right)(\operatorname{Tr} \Delta \Delta) \\
& +\lambda_{5}\left(\Phi^{\dagger} \Phi\right)\left(\eta^{\dagger} \eta\right)+\lambda_{6}\left(\Phi^{\dagger} \Phi\right)\left(\operatorname{Tr} \Delta^{\dagger} \Delta\right)+\lambda_{7}\left(\eta^{\dagger} \eta\right)\left(\operatorname{Tr} \Delta^{\dagger} \Delta\right) \\
& +\lambda_{8}\left(\Phi^{\dagger} \eta\right)\left(\eta^{\dagger} \Phi\right)+\lambda_{9}\left(\Phi^{\dagger} \Delta^{\dagger} \Delta \Phi\right)+\lambda_{10}\left(\eta^{\dagger} \Delta^{\dagger} \Delta \eta\right) \\
& +\mu\left(\eta^{\dagger} \Delta \tilde{\eta}\right)+\text { H.c. }
\end{aligned}
$$

where $\tilde{\eta}=\left(\bar{\eta}^{0},-\eta^{-}\right)$and the parameter $\mu$ has the dimension of mass. Lepton number is then assumed to be broken by explicit soft terms, i.e.

$$
V_{\text {soft }}=\mu_{12}^{2} \Phi^{\dagger} \eta+\mu^{\prime}\left(\Phi^{\dagger} \Delta \tilde{\eta}\right)+\mu^{\prime \prime}\left(\Phi^{\dagger} \Delta \tilde{\Phi}\right)+H . c .
$$


Let $\left\langle\phi^{0}\right\rangle=v_{1},\left\langle\eta^{0}\right\rangle=v_{2}$, and $\left\langle\xi^{0}\right\rangle=v_{3}$, then the minimum of $V$ is given by

$$
\begin{aligned}
V_{\text {min }} & =m_{1}^{2} v_{1}^{2}+m_{2}^{2} v_{2}^{2}+m_{3}^{2} v_{3}^{2}+2 \mu_{12}^{2} v_{1} v_{2} \\
& +\frac{1}{2} \lambda_{1} v_{1}^{4}+\frac{1}{2} \lambda_{2} v_{2}^{4}+\frac{1}{2} \lambda_{3} v_{3}^{4}+\left(\lambda_{5}+\lambda_{8}\right) v_{1}^{2} v_{2}^{2}+\lambda_{6} v_{1}^{2} v_{3}^{2}+\lambda_{7} v_{2}^{2} v_{3}^{2} \\
& +2 \mu v_{2}^{2} v_{3}+2 \mu^{\prime} v_{1} v_{2} v_{3}+2 \mu^{\prime \prime} v_{1}^{2} v_{3},
\end{aligned}
$$

where $\mu_{12}^{2}, \mu, \mu^{\prime}$, and $\mu^{\prime \prime}$ have been assumed real for simplicity. The equations of constraint are obtained from $\partial V_{\min } / \partial v_{i}=0$, i.e.

$$
\begin{aligned}
v_{1}\left[m_{1}^{2}+\lambda_{1} v_{1}^{2}+\left(\lambda_{5}+\lambda_{8}\right) v_{2}^{2}+\lambda_{6} v_{3}^{2}+2 \mu^{\prime \prime} v_{3}\right]+\mu_{12}^{2} v_{2}+\mu^{\prime} v_{2} v_{3} & =0 \\
v_{2}\left[m_{2}^{2}+\lambda_{2} v_{2}^{2}+\left(\lambda_{5}+\lambda_{8}\right) v_{1}^{2}+\lambda_{7} v_{3}^{2}+2 \mu v_{3}\right]+\mu_{12}^{2} v_{1}+\mu^{\prime} v_{1} v_{3} & =0 \\
v_{3}\left[m_{3}^{2}+\lambda_{3} v_{3}^{2}+\lambda_{6} v_{1}^{2}+\lambda_{7} v_{2}^{2}\right]+\mu v_{2}^{2}+\mu^{\prime} v_{1} v_{2}+\mu^{\prime \prime} v_{1}^{2} & =0
\end{aligned}
$$

Consider now the case $m_{1}^{2}<0$, but $m_{2}^{2}>0$ and $m_{3}^{2}>0$ with small $\mu_{12}^{2}, \mu^{\prime}$ and $\mu^{\prime \prime}$. The solutions to the above equations are then

$$
\begin{aligned}
& v_{1}^{2} \simeq-\frac{m_{1}^{2}}{\lambda_{1}} \\
& v_{2} \simeq-\frac{\mu_{12}^{2} v_{1}}{m_{2}^{2}+\left(\lambda_{5}+\lambda_{8}\right) v_{1}^{2}} \\
& v_{3} \simeq-\frac{\mu v_{2}^{2}+\mu^{\prime} v_{1} v_{2}+\mu^{\prime \prime} v_{1}^{2}}{m_{3}^{2}+\lambda_{6} v_{1}^{2}}
\end{aligned}
$$

Since $\mu^{\prime}$ violates $L$ by 1 unit and $\mu^{\prime \prime}$ violates $L$ by 2 units, it is reasonable to assume that $\mu^{\prime \prime} / \mu^{\prime} \sim \mu^{\prime} / \mu \sim v_{2} / v_{1}$. Thus for $m_{2}, m_{3}$, and $\mu$ all approximated by $M \sim 1 \mathrm{TeV}$,

$$
v_{2} \sim \frac{\mu_{12}^{2} v_{1}}{M^{2}}, \quad v_{3} \sim \frac{v_{2}^{2}}{M}
$$

This shows that $v_{3}<<v_{2}<<v_{1}$, and

$$
v_{3} \sim \frac{\mu_{12}^{4} v_{1}^{2}}{M^{5}}
$$

i.e. the analog of Eq. (2). For $v_{1} \sim 10^{2} \mathrm{GeV}$ and $\mu_{12} \sim 1 \mathrm{GeV}$, the solutions are $v_{2} \sim 0.1$ $\mathrm{MeV}$ and $v_{3} \sim 10^{-2} \mathrm{eV}$ as desired. 
In conclusion, a scenario has been presented where a Higgs triplet at the TeV scale is responsible for neutrino masses. The decay of $\xi^{++}$into $l_{i}^{+} l_{j}^{+}$would then map out the neutrino mass matrix, as proposed previously [8]. In addition, a second Higgs doublet at the TeV scale is predicted [9] so that $\xi^{++} \rightarrow \eta^{+} \eta^{+}$is also possible if kinematically allowed.

This work was supported in part by the U. S. Department of Energy under Grant No. DEFG03-94ER40837. 


\section{References}

[1] S. Weinberg, Phys. Rev. Lett. 43, 1566 (1979).

[2] E. Ma, Phys. Rev. Lett. 81, 1171 (1998).

[3] M. Gell-Mann, P. Ramond, and R. Slansky, in Supergravity, edited by P. van Nieuwenhuizen and D. Z. Freedman (North-Holland, Amsterdam, 1979), p. 315; T. Yanagida, in Proceedings of the Workshop on the Unified Theory and the Baryon Number in the Universe, edited by O. Sawada and A. Sugamoto (KEK Report No. 79-18, Tsukuba, Japan, 1979), p. 95; R. N. Mohapatra and G. Senjanovic, Phys. Rev. Lett. 44, 912 (1980).

[4] J. Schechter and J. W. F. Valle, Phys. Rev. D22, 2227 (1980).

[5] E. Ma and U. Sarkar, Phys. Rev. Lett. 80, 5716 (1998); T. Hambye, E. Ma, and U. Sarkar, Nucl. Phys. B602, 23 (2001).

[6] R. Foot, H. Lew, X.-G. He, and G. C. Joshi, Z. Phys. C44, 441 (1989).

[7] E. Ma, hep-ph/0112232 (Mod. Phys. Lett. A, in press).

[8] E. Ma, M. Raidal, and U. Sarkar, Phys. Rev. Lett. 85, 3769 (2000); Nucl. Phys. B615, $313(2001)$.

[9] E. Ma, Phys. Rev. Lett. 86, 2502 (2001). 